\title{
Current over-stressing small DC motors to evaluate performance limits of electromechanical actuators for haptic applications
}

Conference or Workshop Item

Accepted Version

Jarman, R., Janko, B. and Harwin, W. S. (2015) Current overstressing small DC motors to evaluate performance limits of electromechanical actuators for haptic applications. In: IEEE World Haptics Conference, 22-26 Jun 2015, pp. 171-176. doi: https://doi.org/10.1109/WHC.2015.7177709 Available at http://centaur.reading.ac.uk/67692/

It is advisable to refer to the publisher's version if you intend to cite from the work. See Guidance on citing.

Published version at: http://dx.doi.org/10.1109/WHC.2015.7177709

To link to this article DOI: http://dx.doi.org/10.1109/WHC.2015.7177709 
including copyright law. Copyright and IPR is retained by the creators or other copyright holders. Terms and conditions for use of this material are defined in the End User Agreement.

\section{www.reading.ac.uk/centaur}

\section{CentAUR}

Central Archive at the University of Reading

Reading's research outputs online 


\title{
Current over-stressing small DC motors to evaluate performance limits of electromechanical actuators for haptic applications
}

\author{
Rebecca Jarman ${ }^{1}$, Balazs Janko² and William S. Harwin² ${ }^{2}$
}

June 2015

\begin{abstract}
Actuators for haptic devices tend to have a different set of requirements in comparison to many other engineering applications. Small permanent magnet DC electric motors are commonly used as actuators in haptic devices and, in operation, tend to spend a significant period of time in a 'stalled' condition where they are attempting to oppose an applied force. Ideally a haptic actuator together with its power amplifier exchange energy reversibly with the mechanical loads. However this is not feasible at room temperature and to achieve good force performance results in energy loss as heat in the motor windings. This paper identifies the relationship between heat loss and force generation in haptic electromagnetic actuators. The work then presents results on current over-stressing of small DC motors so as to understand the risks of demagnetisation against thermal damage to the armature. Results indicate that it should be possible to apply short current over-stresses to commercial DC permanent magnet motors to increase end point force. Also by paying careful attention to heat dissipation in the design of small permanent magnet actuators motors, it should be possible to improve the overall performance of actuators for haptic applications.
\end{abstract}

\footnotetext{
${ }^{* 1}$ Rebecca Jarman is with Renishaw PLC, UK beckyjbass@gmail.com

$\dagger^{2}$ Balazs Janko and William Harwin are with the School of Systems Engineering, The University of Reading, UK w.s.harwin@reading. ac.uk
}

\section{Introduction}

${ }^{1}$ Magnetic field based actuators are widely used in haptics applications, and due to cost and simplicity these are most likely to use permanent magnets to create the B-field and brushed contacts to switch currents in the armature[1]. In most applications some mechanical gearing is needed to map the high speed, low torque characteristics of the small DC motors to high torque slower loads. When a low loss transmission with minimal backlash is used to transfer loads to the linkage the resulting concept is often termed as 'backdrivable' haptics. Under these circumstances the controller assumes an invertible relationship between motor torque and end point force[2].

Where haptic applications differ from most other applications (with some exceptions in robotics[9, 10]) is in the need to operate the actuator in a 'stalled' condition for significant periods of time. Motor manufacturers do not characterise this operational point well and simply refer to a stall current or stall torque.

A few haptic device designs have chosen to eliminate the mechanical gear reductions and direct drive the linkage, for example Entact Robotics (entactrobotics.com Canada) produces a family of direct drive devices W5D, W3D etc. A similar example was the Logitech Wingman mouse where the actuator was tightly coupled to the linkage as shown in Fig 1. A direct drive approach has two strong advantages: simplifying the design and hence the end unit cost, and

\footnotetext{
${ }^{1}$ Published in IEEE World Haptics Conference (WHC) (June 2015).
} 


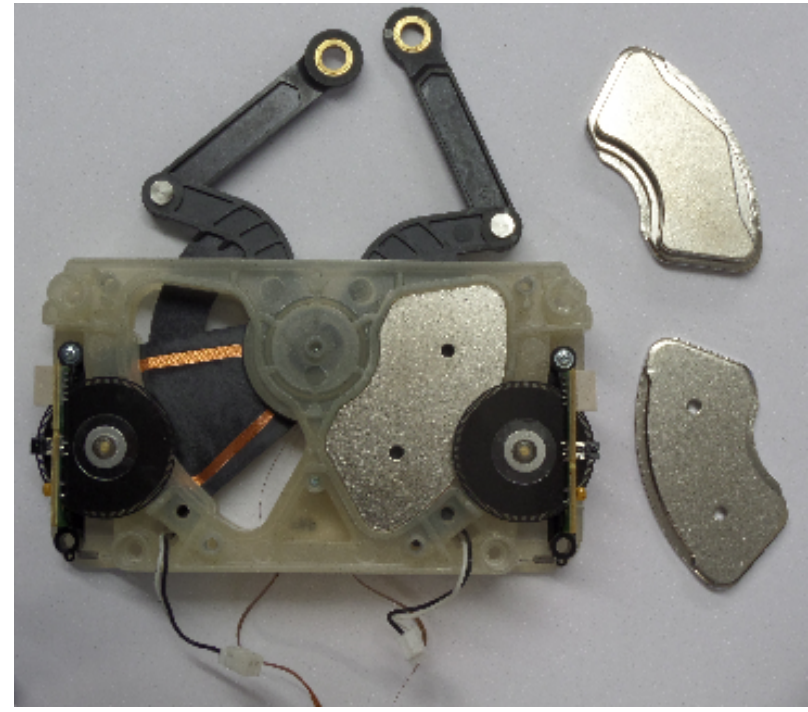

Figure 1: Actuators in a Logitech Wingman Force Feedback Mouse with field magnets removed to expose windings.

reducing the 'free space inertia'. The penalty is the reduction of end point force so the haptic illusion is destroyed by 'push through', likewise controller design becomes harder with fewer opportunities to lose energy in the transmission, a concept covered in the classic paper by Colgate and Schenkel[3]. However a possible benefit of this approach is that the rotation of the motor is limited to a few radians. The purpose of this paper is to argue that these benefits should dictate a re-investigation of small permanent magnet motors for haptic applications given these advantages.

Direct drive implies the need for high currents, and two broad mechanisms of failure can be identified: armature damage as a result of over heating and demagnetization of the permanent magnets. Armature overheating is most often considered in the design phase since the effects tend to be catastrophic whereas motor demagnetization is characterised by loss of performance.

Leuschke et al.[7] discuss the design of a direct drive fingertip haptic display that includes a bespoke designed actuator. They consider a basic heat model but make no attempt to improve performance by considering the thermal design of the armature. In a later paper Buckley et al. [1] describe a thermal model of armature heating and validate it against measurements. The thermal model is then used in the control architecture.

There has also been an investigation into transient thermal analysis of brushed motors for automotive applications [5] that considers the transient loading of DC motors used in ABS systems, windscreen wipers and other such systems to predict life expectancy. The conditions here are more representative of haptic applications, but fall short of being directly comparable.

Research into the demagnetisation of different motor types mainly focuses on the continuous running of larger motors. For example Kim et al.[6] do a finite elements analysis (FEM) on the fields in three types of synchronous motor rotor and Ruoho et al.[8] describe the interdependence of loading, temperature and demagnetisation on permanent magnet motors. Neither paper has much to contribute to haptic actuators other than to highlight design processes.

Thus pertinent questions in haptic actuator design are to understand the conditions leading to failure of a motor under high torque (high current) conditions and evaluate the potential to adapt the design of small permanent magnet motors to better suit the needs of haptic devices.

\section{Heat dissipation in the air- gap}

A relatively common model of a motor can be derived from Maxwell's equations and the Lorenz Force Law. This predicts the motor torque $T$ from the current $i$ as $T \approx K_{T} i$ where $K_{T}$ is a combination of parameters (field strength in the air-gap, number of segments and length of conductor exposed to the field etc.), usually assumed constant for direct current permanent magnet motors. A related equation, sometimes known as the generator equation, relates an internal back e.m.f to the motor speed $\omega$ where $V \approx K_{g} \omega$ and although often published as separate values, these two 
constants are identical. Variations of these two equations can be applied to most electromagnetic actuators, and can be extended to enable models of the driver electronics (such as winding impedance, electronic commutation) and the drive train (friction, efficiency, inertia).

For haptic devices based on a backdrivable linkage, the freespace condition will be compromised primarily by friction and inertia of the mechanical components. A high transmission gear ratio adds significantly to these components. The hard contact condition of a backdrivable linkage is determined primarily by the torque generated by the motor although friction in the transmission may add a small component. Factors that enable a DC actuator to achieve a high torque are armature current, magnetic field in the air-gap, the number of conductors carrying the current and the length of the conductor in the air-gap. These factors also determine the level of heat generated in the air-gap thus for haptic applications, this heat generation and management should be a major factor in actuator design.

Here we consider a classic design of small permanent magnet motors where the air-gap is primarily filled with copper and is thus able to generate a high torque while keeping the inertia low. The model is further simplified by considering only the cross sectional area of the air-gap $A_{g a p}$ and the length $l_{g}$ and assuming the magnetic field does not stray outside this volume.

We can consider an armature with $n$ conductors in this space. If there is a complete filling of the space with the conductor then the average cross sectional area $a$ of a single conductor is $a=A_{\text {gap }} / n$. We can thus estimate the total resistance $R$ of conductor in the air-gap from the definition of resistivity (the Drude model)

$$
\rho=\frac{R A_{\text {gap }} / n}{n l_{l}}
$$

- Exemplar values of $\rho$ at $20^{\circ} \mathrm{C}$ are, for copper $1.72 \times 10^{-8} \Omega m$, for aluminium $2.82 \times 10^{-8} \Omega m$ and for silver $1.587 \times 10^{-8} \Omega m$

Thus the resistance of conductor able to generate a force and hence a torque from the field in the air-gap is

$$
R=\frac{\rho l_{g} n^{2}}{A_{g a p}}
$$

If we now assume an average field strength $B$ in the air-gap we can estimate the force $F$ as $F=B i l_{q} n$, so $i=F / B l_{g} n$. We can now also estimate the $i^{2} R$ power loss in the air-gap. Power lost is

$$
P_{\text {loss }}=i^{2} R=\frac{F^{2}}{\left(B l_{g} n\right)^{2}} \frac{\rho l_{g} n^{2}}{A_{g a p}}=\frac{F^{2} \rho}{B^{2} V_{g a p}}
$$

and is independent of the number of conductors $n$. $V_{\text {gap }}$ is the effective volume of the air-gap, that is $V_{\text {gap }}=A_{\text {gap }} l_{g}$.

This can be expressed as

$$
F=B \sqrt{\frac{V_{g a p}}{\rho}} \sqrt{P_{l o s s}}
$$

This relationship can be related to the endpoint force by considering the transmission gear ratio that would simply scale the force by some value $\alpha=r_{a} / r_{l}$, the armature radius and effective link length respectively. Crucially eqn 2 shows the dependence of the forces reflected to the user on the power loss in the air-gap. Eqn 2 can also be coupled to the definition of the motor torque or generator constant, thus if we assume that the torque constant can be estimated approximately as $K_{T}=B l_{g} n r_{a}$ where $r_{a}$ is the armature radius. We can use eq 1 to show that

$$
B r_{a} \sqrt{\frac{V_{g a p}}{\rho}}=\frac{K_{T}}{\sqrt{R}}=M
$$

Where $M$ is a figure of merit indicating the power loss and hence the ability to generate high torques, and can be computed from the motor specifications. So an actuator's ability to generate end-point forces $F_{\text {end }}$ is proportional (through $\alpha$ ) to the motor characteristics

$$
F_{\text {end }}=\frac{r_{a}}{r_{l}} M \sqrt{P_{\text {loss }}}
$$

Thus the heat generated in the air-gap is a key performance limitation for motors in haptic devices and there are almost certainly gains to be achieved if greater consideration is given to designing the thermodynamic properties of the armature of a haptic 


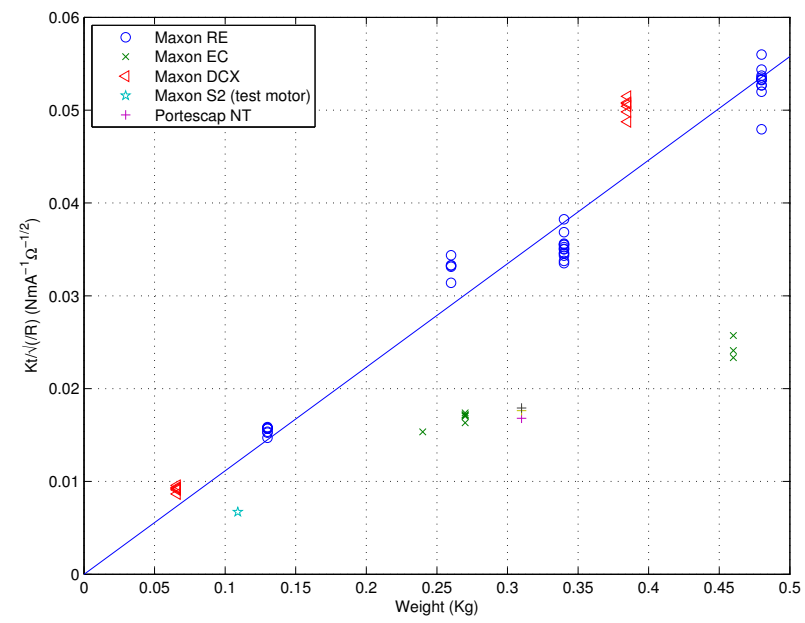

Figure 2: Comparison of some small DC motors from Maxon motors (RE, EC, DCX and S2) and Portescap (NT) with regression line $\frac{K_{T}}{\sqrt{R}}=0.11 w$ (where $w$ is the weight in kilograms)

actuator so heat is removed from the armature. Monitoring or modelling the temperature in the air-gap is also required to provide a safeguard against motor damage although it is likely that additional research will be needed to gain a good understanding of these thermal limits. The risk remains of motor demagnetisation at high armature currents so a set of experiments were devised to evaluate the contributing risks of heat damage and demagnetisation by testing brief current over-stress in a commercial DC motor.

\section{Current over-stressing stalled DC motor}

To evaluate the scope for specialist actuators to be used in haptic applications we tested the effects of current over-stress on a pair of small DC motors. Having observed that the usual cause of failure of motors in haptic applications is heat damage to the armature, an observation consistent with heat generated in the air-gap causing the epoxy in the windings to melt, we wished to evaluate the other potential failure mechanism that would reduce the effectiveness of
Table 1: Specifications of the S2326-934 (Maxon Motors). The motor uses AlNiCo permanent magnets

$\begin{array}{lrl}\text { Nominal voltage } & 9.0 & \mathrm{~V} \\ \text { Max continuous current } & 1.36 & \mathrm{~A} \\ \text { Max (peak) current } & 5.17 & \mathrm{~A} \\ \text { Stall torque } & 45.7 & \mathrm{mNm} \\ \text { Armature Resistance } & 1.74 & \Omega \\ \text { Torque constant } & 8.85 & \mathrm{mNm} / \mathrm{A}\end{array}$

motors in haptic applications, i.e. demagnetisation of the permanent magnets.

The fundamental hypothesis is that better thermal design will allow more heat to be removed from the air-gap resulting in a lower temperature and less chance of armature failure. However to ensure that this is a useful design goal, we should ensure that other forms of irreversible damage do not restrict performance improvements. Motor manufacturers often quote a stall current for their products but without any clear explanation. It is possible that stall currents might create a magnetic field sufficiently large to cause demagnetisation of the permanent magnets and the purpose of these experiments is to determine if this is a realistic limitation.

\subsection{Experimental set up}

Two small DC motors were obtained for these experiments under the Maxon Motors old stock program. The motors were a pair of $26 \mathrm{~mm}$ diameter, S2326934 6W DC brushed motors with AlNiCo permanent magnets and graphite brushes (see Table II). A test rig shown in Fig 3 (upper) held the motors such that torque at stall could be measured via two $750 \mathrm{~g}$ load cells (CZL639HD-750g, Dongguan South China Sea Electronic Company Ltd).

The overload testing involved charging the capacitor to voltages between $12 \mathrm{~V}$ and $68 \mathrm{~V}$ then discharging it across the motor under test resulting in currents of up to 43 amps (8 times the manufacturer's limit). During discharge the forces, and hence the torque, were measured in the load cells. The capacitor voltage and current along with the resulting output force were recorded using xPC target and a Quanser Q8 

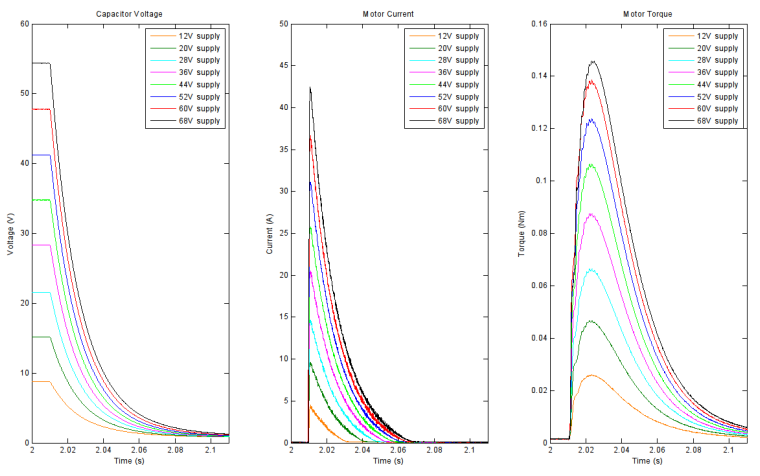

Figure 5: Motor B: Run 1 Discharge graphs. (Left) Voltage across $11 \mathrm{mF}$ capacitor. (Centre) Current through the motor. (Right) Measured motor torque during discharge (right)

Hardware-In-Loop (H.I.L) system which also generated the trigger pulse to discharge the capacitor. The electronics are shown in Fig 3 (lower).

Prior to the experiments the load cells and their associated amplifiers were calibrated via a series of small weights between 1 and $600 \mathrm{~g}$. Further details of the test rig, electronics and calibration process are given in Jarman[4].

\section{$3.2 \quad$ Results}

Following a commissioning phase the two motors were tested. Motor A was tested through an initial (demagnetising) run and two further runs. Motor B was tested through an initial and a single follow up run.

Each run consisted of

1. Measuring and recording the motor torque constant and armature resistance

2. Charging the capacitor to $12 \mathrm{~V}$ and performing the first over-stress discharge of the motor

3. Measure and record the motor torque constant and armature resistance

4. Repeat steps 2 and 3 for capacitor voltages of $20,28,36,44,52,60$ and 68 volts.

Between each discharge the motor torque constant and armature resistance were measured by initially

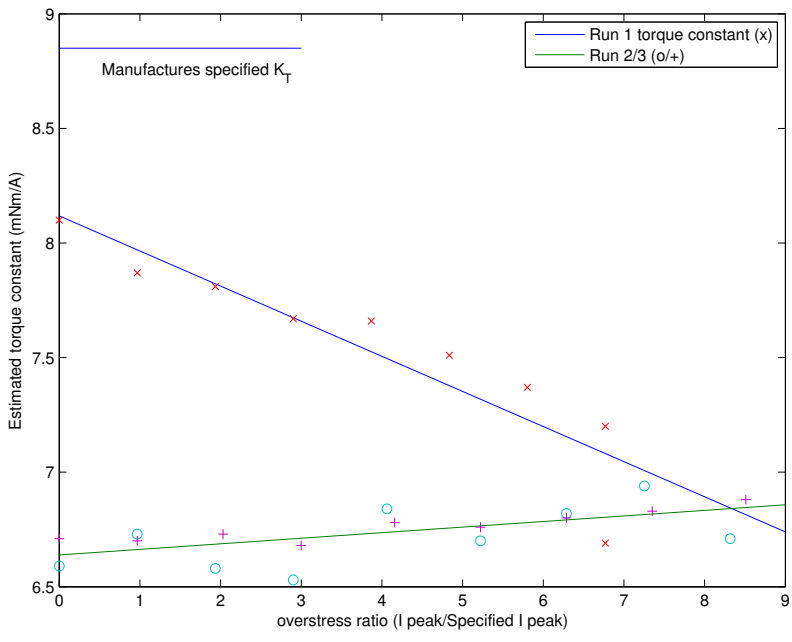

Figure 6: Motor A: Change of motor torque constant with progressive current over-stressing. Overstressing factor is with respect to the manufacturer's specified Max. (stall) current of $5.17 \mathrm{~A}$, thus peak currents were approximately $35 \mathrm{~A}$. Measured values of $K_{T}$ were consistently lower than that specified by the Manufacturer.

setting the motor voltage to $2 \mathrm{~V}$ and measuring the current and torque. This voltage was then reduced in 0.25 volt increments so as to ensure the motor made good initial contact with the load cells, the current was well within the maximum specified current of $1.15 \mathrm{~A}$ and the motor could not be overloaded. The motor current and the output of both channels of the strain gauge amplifier were recorded against each input voltage and the process was repeated 3 times to ensure a good estimate of measurement errors.

A typical set of discharge results is shown in Fig 4. The capacitor voltage and motor current are well correlated and consistent with the armature resistance plus sense resistor (10 milliohms). The torque discharge curve shows a double first order response with time constants believed to be of the order $1 / 65$ seconds (current discharge), and 1/90 seconds representing the mechanical coupling of the motor to the torque sensor and the filtering in the strain gauge sensor.

The motor specification measurements were made 


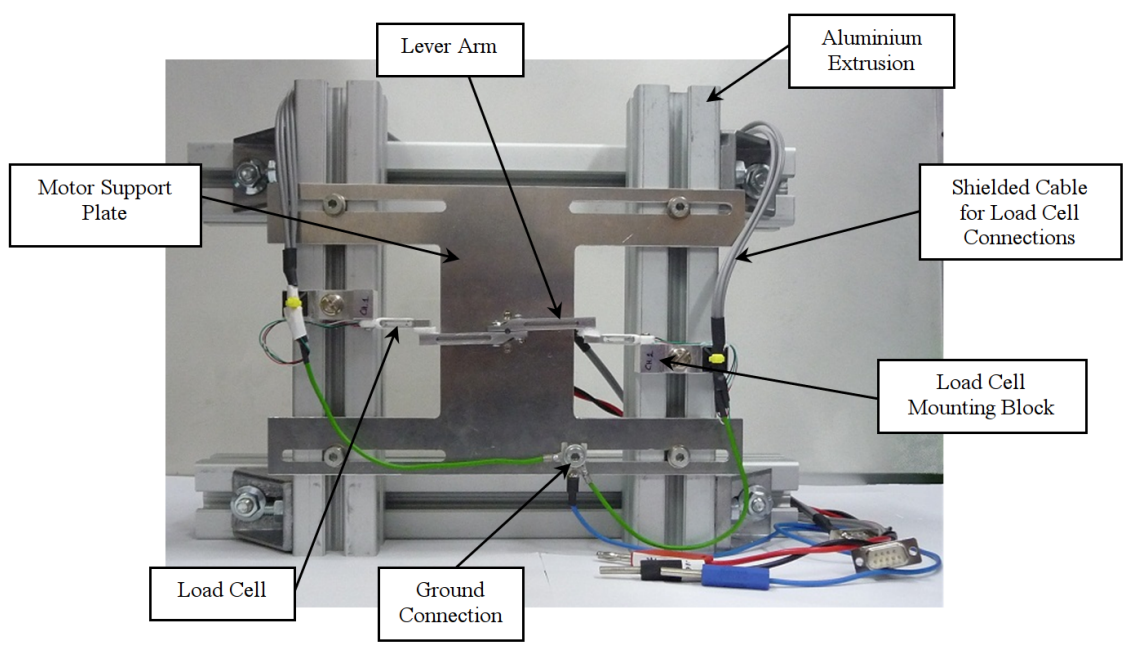

Figure 3: Test rig. The motor under test is behind the motor support plate. Load cells are shown attached to the lever arms.

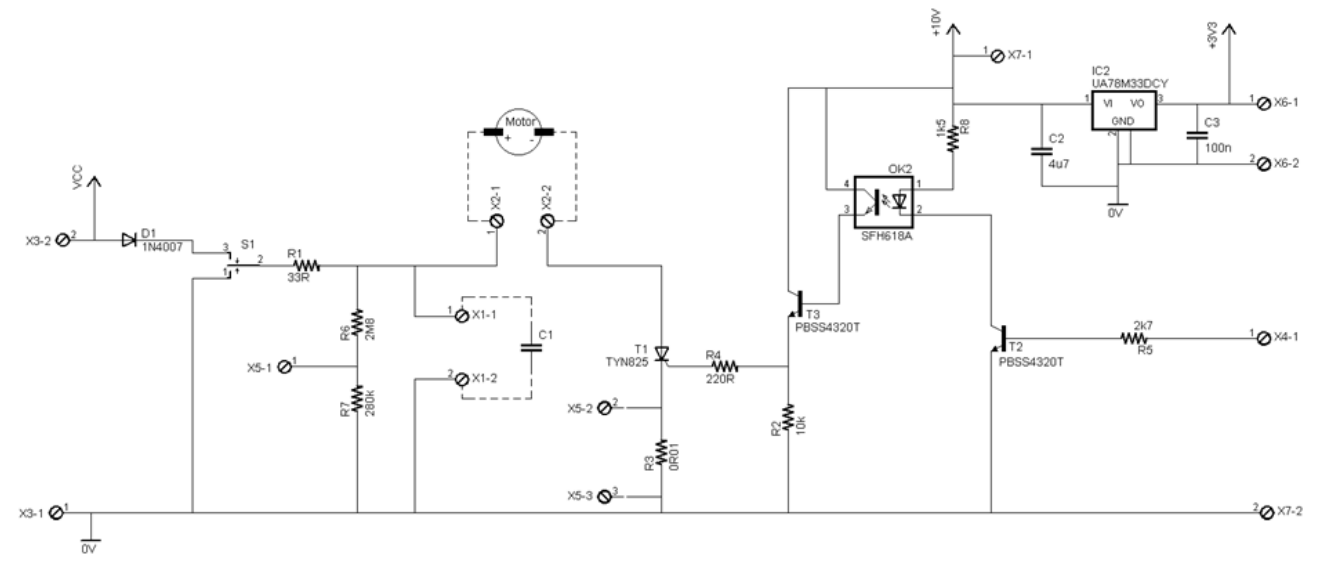

Figure 4: Test electronic circuit showing SCR discharge circuit 


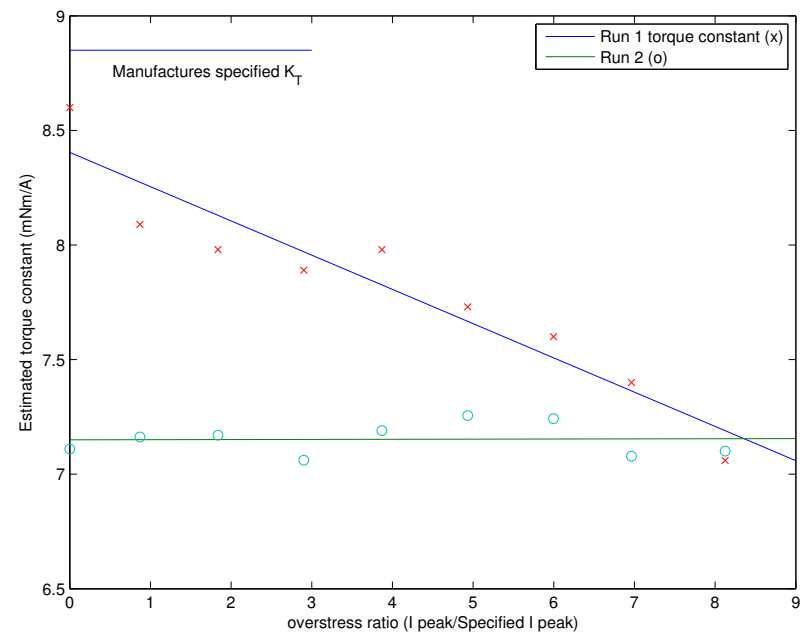

Figure 7: Motor B: Change in motor torque constant with progressive current over-stressing. See also Fig 5

between each discharge. Fig 5 and Fig 6 show the changes to the torque constant for each of the motors following each discharge. The horizontal axis is the over-stress factor, calculated as the ratio of the peak current during discharge over the motors specified maximum peak current (5.17 amps in the case of the tested motors). For both motors there is a reduction in the value of the torque constant during the first set of over-stress experiments. Both motors appear to show a slight recovery of torque constant in subsequent runs. It is not clear if this is an artefact of the experiment or an unexplained process, but it is not unreasonable to conclude that there is no further degradation of the torque constant with similar levels of over-stressing.

\subsection{Discussion}

The results show that it is possible to apply a significant (approximately eight times) transient overload to a DC brushed motor to provide a substantial increase in output torque at the expense of a small reduction in the continuous torque.

The demagnetisation effects appear to stabilise after the first overload providing repeatable performance for a given maximum overload. There were no obvious effects of temperature on the motor performance during the tests. Tests were done in at room temperature, and although slight warming of the casing was observed it was not measured. It should be noted that the motors in these tests were based on AlNiCo magnets which are more susceptible to demagnetization when compared to motors using Neodymium magnets.

Electromagnetic interference is likely to need greater attention since higher currents will increase the probability of brush arching as currents are switched across segments. Without further investigation it is difficult to recommend the extent of this problem or to suggest using electronic commutation as an alternative.

Current over-stressing events were relatively short so as to avoid heating effects and it is possible that over-stressing the motor for longer time periods might have greater long term effects on demagnetization and there may be other impacts on the actuator life.

Thus the results give evidence that, in haptic applications, current over-stressing may enable greater performance from commercial permanent magnet DC motors designed for general applications. Current over-stressing is particularly appropriate for arresting collisions since a short, high force essentially acts as a force impulse. The technique will be less useful for continuous contact where the actuator must run under stall conditions for longer periods of time.

Heat generated in the air-gap is likely to remain the biggest problem in the design of actuators specifically for haptic applications. The model outlined above indicates that the available force will be dictated by this heat loss although it may be possible to have some influence by adjusting the airgap volume, the size of the armature and the fields produced by the permanent magnets. However the most fruitful approach is likely to be to transfer heat away from the air-gap to allow greater currents to flow in the windings without risking thermal damage to the armature. Forced fluid flow through the air-gap is an option and both air and liquids may be appropriate. A problem with liquid cooling is that of containing the liquid which either must be contained by the motor bearings, or kept in a closed heating system. A 
better prospect is to conduct the heat out of the airgap using the copper conductors, and then using a coolant or efficient radiation to then pump the heat away. To assess this approach further will require detailed thermal models of the armature, validated by experimental prototypes. It may well be that the thermal design can benefit from the fact that, for a haptic device, the actuator needs to move only by a few radians, and it may be possible to use forced fluid cooling through flexible connectors, rather than require sealed bearings. Lowering the temperature of the windings will also be beneficial since it reduces the resistivity of copper. It may be that eventually superconducting materials can be designed to allow higher currents at an acceptable temperature, but with materials currently available there remain major problems in insulating the superconducing magnets from the user.

Other design factors relevant to EM actuators designed for haptic applications would be the benefit of integrating a sensor to measure the air-gap temperature. This simple expedient would enable the control system to manage heat as part of the control design without the need to rely on a thermal model. Similarly most haptic devices need accurate position measurement at the motor shaft and this could also be built into the motor. In most cases designers of haptic interfaces simply wish to command a torque based on position so there may even be potential for low level controllers to be built into the actuator.

\section{Conclusion}

For haptic interfaces based on the concept of a backdrivable linkage and using small DC permanent magnet motors, better performance would be achieved if designers and research engineers allowed current over-stressing of the actuators under carefully managed conditions. Evidence is given that shows that in return for a small loss in performance, larger forces could be exerted by the commercially available DC motors provided by exceeding the manufacturer's specified current for short periods of time (current over-stressing).

This paper also argues for new designs of actuators for haptic applications. Two limitations are of particular concern, demagnetization of the permanent magnet, and damage to the actuator due to excessive heat generated in the air-gap. A model is given that shows the relationship of this heat loss to the ability of the actuator to generate force. The force produced by the actuator, and hence the haptic device, is proportional to the square-root of the power lost in the armature. This model provides a figure of merit that can be used to compare commercial actuators. A small amount of demagnetisation is evident from the current over-stressing for the AlNiCo motors used in these experiments, but the effect is only observed on the first run, thereafter the motor specification remains constant thus indicating good potential performance gains.

\section{Acknowledgements}

The authors are grateful for the help from Peter Tolson with the construction of the test-rig, and also Maxon motors and in particular Shashi Mistry for access to 'old stock' motors.

\section{References}

[1] P. Buckley, H.H. King, M. Wang, and B. Hannaford. Effects of thermal protection methods on haptic perception. In World Haptics Conference (WHC), 2011 IEEE, pages 143-148, June 2011.

[2] C.R. Carignan and K.R. Cleary. Closed-loop force control for haptic simulation of virtual environments. haptics-e.org, 1(2), February 2000.

[3] J.E. Colgate and G. Schenkel. Passivity of a class of sampled-data systems: Application to haptic interfaces. In American Control Conference, pages 3236-3240, 1994.

[4] Rebecca Jarman. Over-stressing dc electric motors for haptic applications. Master's thesis, The University of Reading, School of Systems Engineering, September 2011. 
[5] Jacek Junak, Grzegorz Ombach, and Dave Staton. Permanent magnet dc motor brush transient thermal analysis. In Proceedings of the 2008 International Conference on Electrical Machines, pages 978-1, 2008.

[6] Ki-Chan Kim, Kwangsoo Kim, Hee-jun Kim, and Ju Lee. Demagnetization analysis of permanent magnets according to rotor types of interior permanent magnet synchronous motor. Magnetics, IEEE Transactions on, 45(6):2799-2802, 2009 .

[7] Rainer Leuschke, Elizabeth KT Kurihara, Jesse Dosher, and Blake Hannaford. High fidelity multi finger haptic display. In Eurohaptics Conference, 2005 and Symposium on Haptic Interfaces for Virtual Environment and Teleoperator Systems, 2005. World Haptics 2005. First Joint, pages 606-608. IEEE, 2005.

[8] Sami Ruoho, Jere Kolehmainen, Jouni Ikaheimo, and Antero Arkkio. Interdependence of demagnetization, loading, and temperature rise in a permanent-magnet synchronous motor. Magnetics, IEEE Transactions on, 46(3):949-953, 2010.

[9] R. Thomas and W.S. Harwin. Estimation of contact forces in a backdrivable linkage for cognitive robot research. In Towards Autonomous Robotic Systems, pages 235-246. Springer Berlin Heidelberg, 2014.

[10] M. Zinn, O. Khatib, B. Roth, and J. Salisbury. A new actuation concept for human-friendly robot design: Playing it safe. IEEE Robotics \& $\mathrm{Au}$ tomation Magazine, pages 12-21, June 2004. 